Georgia State University

ScholarWorks @ Georgia State University

\title{
Variable-frequency-train stimulation of skeletal muscle after spinal cord injury
}

\author{
C. Scott Bickel \\ University of Alabama - Birmingham, bickel@uab.edu \\ Jill Slade \\ Michigan State University, Erik.Shapiro@rad.msu.edu \\ Leslie R. VanHiel \\ Gordon L. Warren \\ Georgia State University, gwarren@gsu.edu \\ Gary A. Dudley
}

Follow this and additional works at: https://scholarworks.gsu.edu/pt_facpub

Part of the Physical Therapy Commons

\section{Recommended Citation}

Bickel, C.S., J.M. Slade, L.R. VanHiel, G.L. Warren, and G.A. Dudley. Variable frequency train stimulation of skeletal muscle after spinal cord injury. J. Rehabil. Res. Dev. 41: 33-40, 2004.

This Article is brought to you for free and open access by the Department of Physical Therapy at ScholarWorks @ Georgia State University. It has been accepted for inclusion in Physical Therapy Faculty Publications by an authorized administrator of ScholarWorks @ Georgia State University. For more information, please contact scholarworks@gsu.edu. 
JRRD

Joumal of Rehabilitation Research and Developenent

Volume 41 Number 1, January/February 2004

Pages $33-40$

\title{
Variable-frequency-train stimulation of skeletal muscle after spinal cord injury
}

\author{
C. Scott Bickel, PT, PhD; Jill M. Slade, PhD; Leslie R. VanHiel, MSPT; Gordon \\ L. Warren, PhD; \\ Gary A. Dudley, PhD
}

Department of Exercise Science, University of Georgia, Athens, GA; Department of Physical Therapy, Georgia State University, Atlanta, GA; Crawford Research Institute, Shepherd Center, Atlanta, GA

\begin{abstract}
Skeletal muscle, after spinal cord injury (SCI), becomes highly susceptible to fatigue. Variable-frequency trains (VFTs) enhance force in fatigued human skeletal muscle of able-bodied (AB) individuals. VFTs do this by taking advantage of the "catch-like" property of skeletal muscle. However, mechanisms responsible for fatigue in $\mathrm{AB}$ and SCI subjects may not be the same, and the efficacy of VFT stimulation after SCI is unknown. Accordingly, we tested the hypothesis that VFT stimulation would augment torque-time integral in SCI subjects. The quadriceps femoris muscle was stimulated with constant frequency trains (CFTs) (six $200 \mathrm{~s}$ square wave pulses separated by 70 $\mathrm{ms}$ ) or VFTs (a train identical to the CFT, except that the first two pulses were separated by $5 \mathrm{~ms}$ ) in SCI and AB subjects. After 180 contractions ( $50 \%$ duty cycle), isometric peak torque decreased 44, 56, and 67 percent, in the $\mathrm{AB}$ $(n=10)$, acute SCI $(n=10)$, and chronic SCI $(n=12)$ groups, respectively. In fatigued muscle, VFTs enhanced the torque-time integral by 18 percent in $\mathrm{AB}$ subjects and 6 percent in chronic SCI patients, and had no effect in acute SCI patients when compared to the corresponding CFT. The much faster rise times in SCI subjects $(\sim 80 \mathrm{~ms}$ vs. 120 $\mathrm{ms}$ in $\mathrm{AB}$ subjects) probably contributed to the inability of VFTs to enhance torque-time integrals in SCI patients. The results suggest that the use of VFT stimulation in patients with SCI may not be as efficacious as it is in AB persons.

Key words: catch-like property, electrical stimulation, fatigue, spinal cord injury, variable frequency train
\end{abstract}

Abbreviations: $\mathrm{AB}=$ able-bodied, $\mathrm{A} / \mathrm{D}=$ analog/digital, $\mathrm{CFT}=$ constant-frequency train, $\mathrm{IPI}=$ interpulse interval, $\mathrm{m} . \mathrm{QF}=$ quadriceps femoris muscle, $\mathrm{MVC}=$ maximum voluntary contraction, $\mathrm{SCI}=$ spinal cord injury, $\mathrm{T} 20-80=$ time from 20 to 80 percent of peak torque, VFT = variable-frequency train.

This material was based on work supported by the Shepherd Center, the Foundation for Physical Therapy, and the National Institutes of Health (HD 37439-S1 and HD 39676).

Address all correspondence and requests for reprints to C. Scott Bickel, PT, PhD; Department of Physical Therapy, Louisiana State University Health Sciences Center, 1900 Gravier Street, New Orleans, LA 70112; 504-568-4288; fax: 504-568-6552; email: cbicke@1suhsc.edu.

\section{INTRODUCTION}

Affected skeletal muscle, after spinal cord injury (SCI), has a reduced ability to generate and maintain force. Significant muscle atrophy and conversion to a fast fiber composition with low levels of oxidative enzymes has been reported to contribute to the muscle's compromised performance after SCI [1-3]. However, Castro et al. has reported increased fatigability after SCI unrelated to 
changes in metabolic enzymes associated with adenosine triphosphate (ATP) synthesis [4]. Electrical stimulation is often used in the rehabilitative setting for training of paralyzed muscle. However, activation with electrical stimulation will inherently cause more fatigue than contractions of voluntary effort [5]. This is probably due to the repetitive, synchronous stimulation of fast and slow motor units during electrical stimulation in apparent disregard for the size principle regarding the orderly recruitment of motor units, as well as the inability to recruit additional motor units to offset fatigue $[5,6]$. Thus, the ability of a patient with $\mathrm{SCI}$ to do multiple sets or repetitions of activities is compromised because of (1) the nature of the muscle itself and (2) the means of activation.

In light of this compromised ability, developing a means to counter force loss during electrical stimulation of paralyzed muscle would be beneficial. Variable-frequency train (VFT) stimulation has received considerable interest for countering fatigue in skeletal muscle of able-bodied $(A B)$ individuals [7-10]. VFTs take advantage of the "catch-like" property of skeletal muscle by varying the frequency within a train of stimulation [11,12]. Short, then long, inter-pulse intervals (IPI) within a stimulation train enhance the rate of rise in torque, as well as peak torque, and therefore the torquetime integral, by as much as 30 percent in fatigued muscle. Binder-Macleod and colleagues have previously determined that the optimal train to enhance isometric force-time integrals of fatigued muscle is to use a single, brief (5 ms) IPI at the beginning of a constant frequency train (CFT) [7].

The presence of an initial, brief IPI has been reported to occur during voluntary activation of human motor units [13-15]. Therefore, the constant frequency pattern of stimuli that is used during conventional electrical stimulation may not be similar to what occurs under voluntary conditions. This difference may also be amplified in fatigued muscle, as Griffin et al. have reported that the prevalence of initial, brief IPIs becomes more common as the muscle fatigues [15]. Thus, VFT stimulation may be more similar to voluntary motor unit activation with regard to pulse frequency than is CFT stimulation, which is typically used clinically. It should also be noted that despite VFT stimulation having similar pulse frequencies to voluntary motor control, the method of activation is still quite different, because electrically stimulated motor unit activation does not adhere to the size principle.

The above issues raise the question of whether VFT stimulation can augment torque in subjects with $\mathrm{SCl}$, a patient population that could benefit from an optimal stimulation pattern. Accordingly, we tested the hypothesis that VFT stimulation would enhance the torque-time integral in patients with complete SCl. Subjects, either months or years after SCI, had their quadriceps femoris muscle (m. QF) subjected to electrical stimulation with both VFT and CFT stimulation before and after a fatigue protocol. Because the muscles of $\mathrm{SCl}$ subjects produce very low levels of force as compared to $A B$ individuals due to the tremendous muscle atrophy [16], we subjected $A B$ subjects to the same protocol starting with similar initial peak torques.

\section{METHODS}

$\mathrm{SCl}$ patients were screened before participation to ensure that their $\mathrm{m}$. QF could elicit modest force from the electrical stimulation without the occurrence of simultaneous muscle spasms that would interfere with the testing trains. Twenty-two SCI subjects completed all phases of the tests without complications. The SCl subjects were further divided into two groups, acute (injured $<1$ year) and chronic (injured $>1$ year). The acute SCI group (SCI-A) included 10 subjects ( $28 \pm 2 \mathrm{yr}, 177 \pm 4$ $\mathrm{cm}, 77 \pm 6 \mathrm{~kg}, 1$ female, mean $\pm \mathrm{SD}$ ) with their level of injury ranging from C3 to T8 and the average weeks post-injury was $23 \pm 5$. The chronic SCI group (SCI-C) included 12 subjects (36 \pm 6 yr, $180 \pm$ $2 \mathrm{~cm}, 78 \pm 4 \mathrm{~kg}, 2$ females) on average $8 \pm 2$ years post-injury, with the level of injury ranging from C6 to T9. An AB control group of 10 subjects was also studied ( $26 \pm 1 \mathrm{yr}, 175 \pm 3 \mathrm{~cm}, 83 \pm 7 \mathrm{~kg}, 1$ female). Subjects had no history of lower limb pathology and gave informed consent before testing. 
The methods were approved by the Institutional Review Boards of both the University of Georgia and Shepherd Center.

\section{QF Experimental Setup}

The m. QF was studied during stimulated isometric contractions essentially as described previously by others $[5,9,10,16-22]$. Briefly, subjects were seated in a custom-built chair, with the left hip and knee secured at approximately $90^{\circ}$ of flexion. The leg was firmly secured to a rigid lever arm with an inelastic strap to ensure that m. QF would perform only isometric contractions. The moment arm was established by placing a Rice Lake 2000 A load cell (Rice Lake Weighing Systems, West Coleman Street, Rice Lake, WI, USA) parallel to the line of pull and perpendicular to the lever arm. Two $8 \times 10 \mathrm{~cm}$ surface electrodes (Uni-Patch, PO Box 1271, 1313 West Grant Boulevard, Wabasha, MN, USA) were placed on the distal $\mathrm{m}$. vastus medialis and the proximal $\mathrm{m}$. vastus lateralis to allow sufficient recruitment of m. QF, as done previously [5,10].

\section{Electrical Stimulation and Force Recordings}

A constant-current electrical stimulator (model DS7AH, Digitimer Limited, 37 Hydeway, Welwyn Garden City, Hertfordshire, AL7 3BE, England) was triggered with a personal computer using an analog/digital (A/D) board (model KPCI 3108, Keithley Instruments, 28775 Aurora Road, Cleveland $\mathrm{OH}, \mathrm{USA}$ ) and a customized program written with TestPoint software (Version 4.0, Capital Equipment Corporation, 900 Middlesex Turnpike, Billerica, MA, USA). The stimulator delivered six 200 s square wave pulses with either a CFT or a VFT. The CFT consisted of six pulses separated by a $70 \mathrm{~ms}$ IPI, while the VFT had a $5 \mathrm{~ms}$ IPI between the first and second pulses, followed by four additional pulses separated by 70 ms IPIs. The VFT using only one brief IPI was chosen because it has been reported to augment force and produce the greatest force-time integrals in fatigued human skeletal muscle [7]. Torque from the load cell was sampled at $10 \mathrm{kHz}$ by computer using the A/D board.

\section{Experimental Procedure}

The m. QF of each subject was investigated. The current necessary to elicit $~ 25$ percent of the $\mathrm{SCl}$ subjects' estimated maximum voluntary contraction (MVC) was determined. Estimated MVC for each SCl subject was set as the torque equal to 1.3 times body weight, because maximal voluntary torque for knee extension approximates 130 percent of body weight in $A B$ individuals $[5,20,22]$. If 25 percent of the subject's estimated MVC could not be attained, the maximum amount of torque that could be evoked was used. AB subjects were tested after all SCI subjects completed the study, with the goal of matching force levels with the acute SCI group, as their muscles would be expected to be similar in fiber composition [4]. Subsequently, m. QF was potentiated with six-pulse CFTs that were delivered one every $5 \mathrm{~s}$ until force plateaued. When the muscle was highly potentiated, a CFT and a VFT were delivered. The m. QF was then fatigued using 180 six-pulse CFTs delivered at a 50percent duty cycle, which results in substantial fatigue in both $\mathrm{SCl}$ and $\mathrm{AB}$ subjects. Immediately following the 180th train, a CFT and a VFT were delivered in random order.

Torque-time integral, peak torque, and the time from 20 to 80 percent of peak torque (T20-80) were determined from the torque recordings. A $2 \times 2$ time (pre-/post-fatigue) by train type (CFT/NFT) repeated measures analysis of variance was run on each variable using SPSS (Version 10.0). Gain scores were calculated for the percent augmentation between the post-fatigue trains (VFT vs. CFT) to determine if group or train type differences existed. For all statistical tests, $\alpha=0.05$. Cohen's $d$ was calculated as a measure of the post-fatigue effect size of VFTs [23].

\section{RESULTS}




\section{Muscle Fatigue}

Stimulation prior to fatigue resulted in similar initial torques for all three groups (Figure 1, $p>$ $0.05)$. The relative torque decline during the 180 CFT contraction protocol showed group differences, with $\mathrm{AB}$ showing less fatigue than the chronic $\mathrm{SCl}$ group $(p<0.05)$. The trend for the groups was that $\mathrm{AB}$ fatigue was less than the SCl-A, followed by the SCI-C: 44, 56, and 67 percent, respectively. 

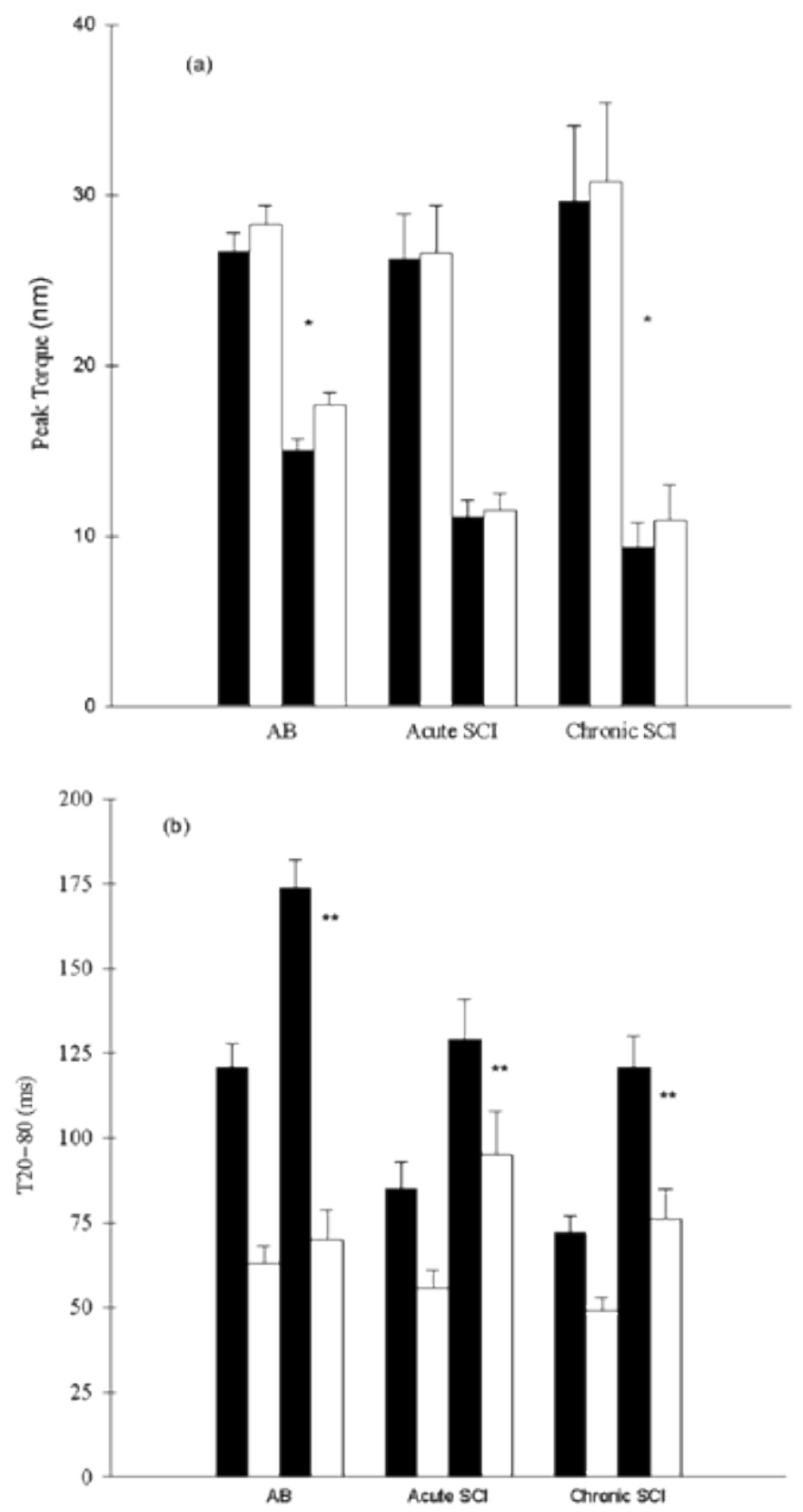

Figure 1.

(a) Peak torque and (b) time from 20 to 80 percent of peak torque (T20-80) for pre- and post-fatigue trains in all three groups. Filled bars represent constant-frequency trains (CFTs) and open bars are variable-frequency trains (VFTs). $A B$ group had significant time $\times$ train type interaction for peak torque and T20-80. Post-fatigue peak torque: *VFT $>$ CFT $(p<0.05)$; * ${ }^{*}$ CFT is significantly slower than VFT.

\section{Torque-Time Integral}


All groups showed significant time-by-train interactions for the torque-time integral $(p<0.05)$. In the $A B$ group prior to fatigue, the CFT torque-time integral was 3 percent greater than that for the VFT, but after the fatigue protocol, the VFT torque-time integral was 17 percent greater (Cohen's $d=$ 0.8 [23]), even though the VFT is 65 ms shorter in duration (Figure 2). In contrast, the CFT had higher torque-time integrals, 10 and 6 percent (Cohen's $d=0.2$ ) for both the pre- and post-fatigue conditions, respectively, for the SCI-A group. The SCI-C group demonstrated a 12 percent greater torque-time integral for the CFT when compared to the VFT prior to fatigue, but after fatigue the VFT enhanced the torque-time integral by 6 percent $(p>0.05$, Cohen's $d=0.1)$ (Figure 2). 

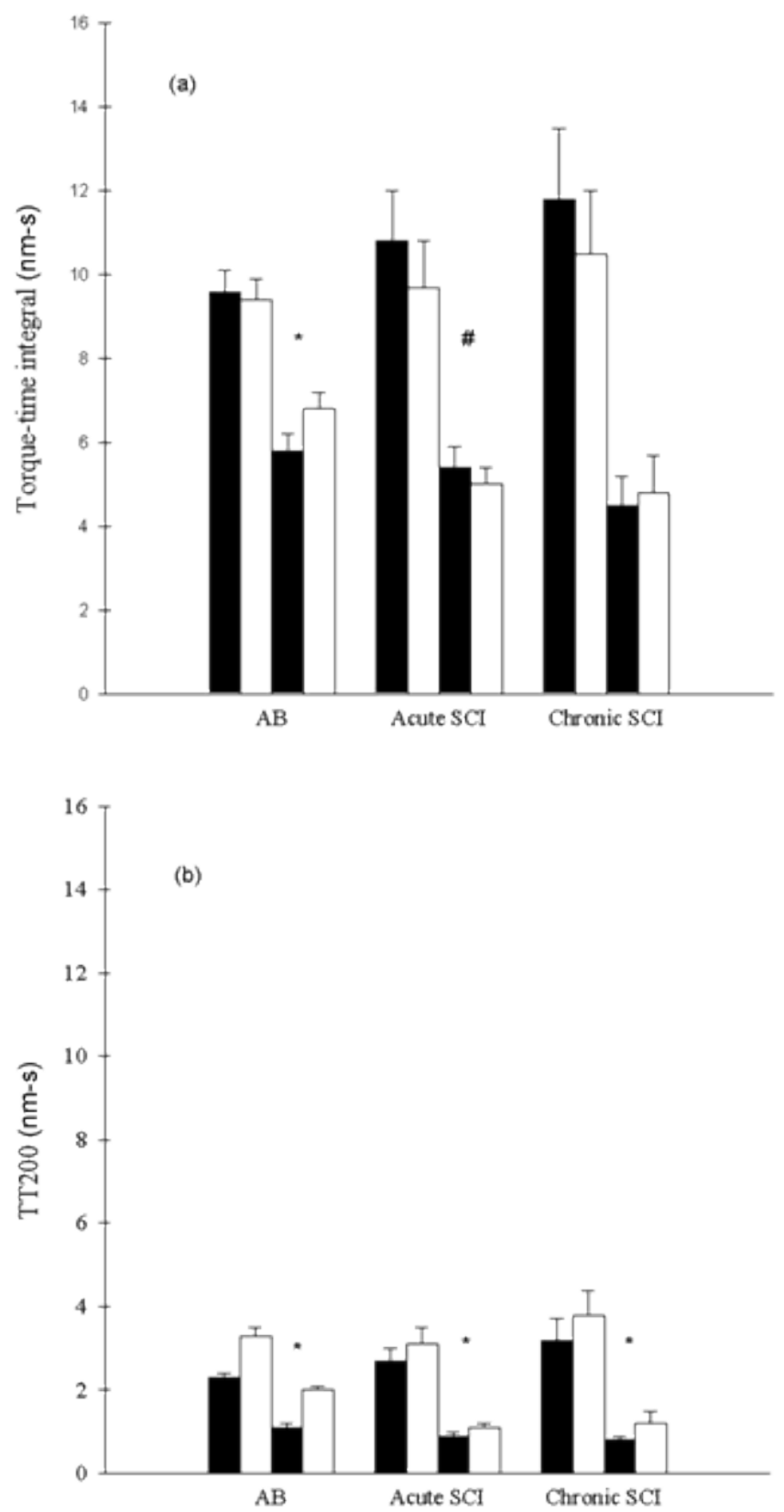

Figure 2.

(a) Torque-time integral and (b) torque-time integral over first $200 \mathrm{~ms}$ (TT200) for pre- and post-fatigue trains in all three groups. Filled bars represent constant-frequency trains (CFTs) and open bars are variablefrequency trains (VFTs). All groups had significant time $\times$ train type interactions for torque-time integral. Post-fatigue: *VFT $>$ CFT and \#CFT > VFT $(p<0.05)$.

\section{Peak Torque}


Greater peak torque can contribute to the augmented torque-time integral that is evoked by VFT stimulation in fatigued muscles (Figure 3). Peak torque responses to CFT and VFT stimulation were different among groups (Figure 1). The $A B$ group had a significant time-by-train interaction for peak torque $(p<0.05)$, with the VFT eliciting a peak torque 18 percent greater than that for the CFT in the fatigued $\mathrm{m}$. QF. The SCI-A group showed neither a time-by-train interaction $(p>0.05)$ nor a main effect for train $(p>0.05)$, indicating that the type of train did not affect peak torque. The SCI-C group did not show a time-by-train interaction $(p>0.05)$, but did have main effects for type of train and time, indicating that the train effect (higher peak torque for VFT) was the same both pre- and postfatigue. 

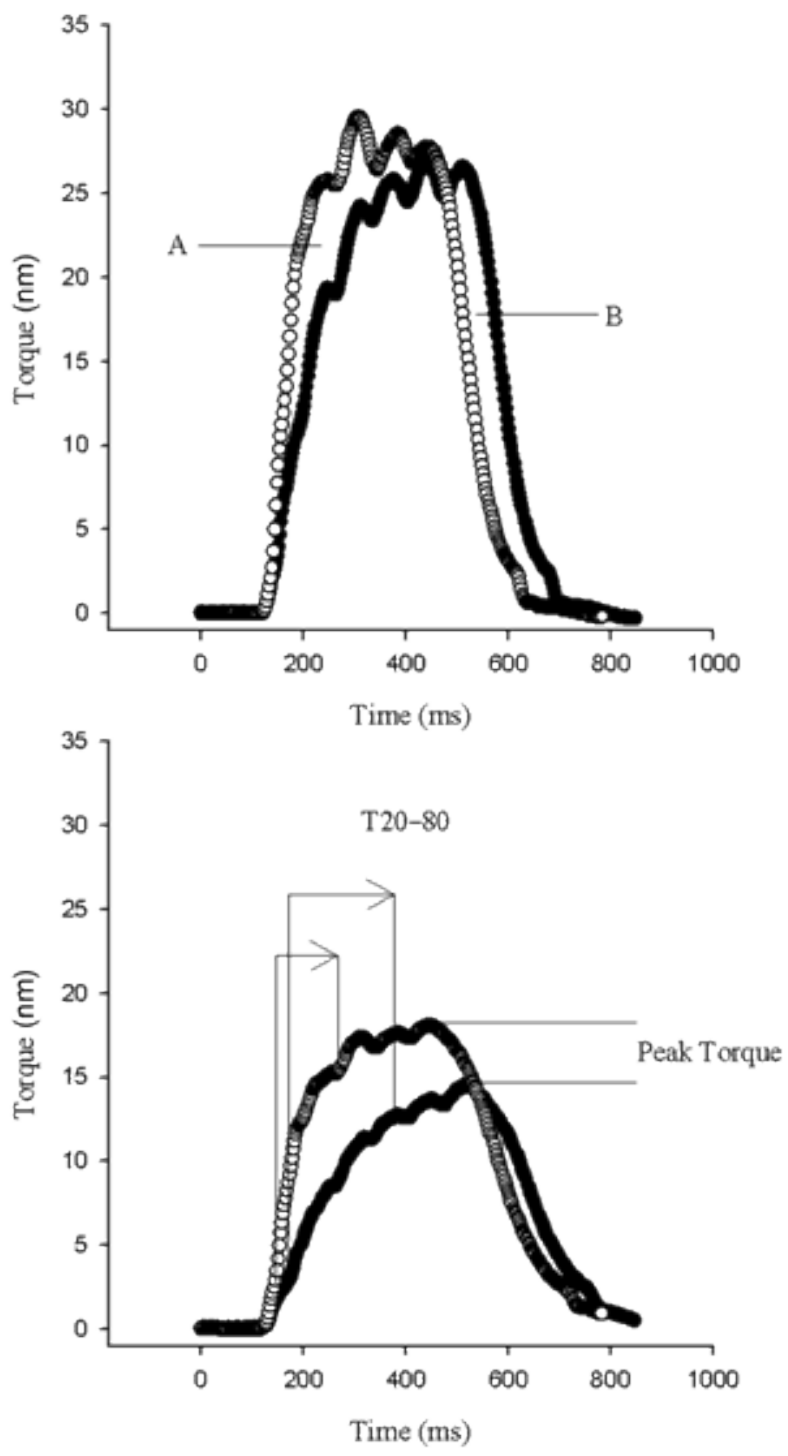

Figure 3 .

Representative torque tracings from able-bodied subject for variablefrequency trains (VFTs) (open circles) and constant-frequency trains. (CFTs) (closed circles) stimulation pre- (upper panel) and post(lower panel) fatigue. Note that area $\mathrm{A}$ and $\mathrm{B}$ are approximately same size in upper panel, whereas in lower panel, corresponding A and B regions are markedly different. Torque-time integral (area under curve) is increased by either reducing time from 20 to 80 percent of peak torque (T20-80) and/or increasing peak torque.

\section{Time from 20 to 80 Percent of Peak Torque}

Another factor that can contribute to an enhanced torque-time integral by VFT stimulation in fatigued muscle is a more rapid rise time (Figure 3). The T20-80 for the AB group had a significant time-by-train interaction. 
The CFT T20-80 was 44 percent slower after the fatigue protocol when compared to the pre-CFT T20-80, while the VFT T20-80 slowed by only 11 percent with fatigue (Figure 1). The fact that the CFT showed such increased slowing of contraction compared to VFT probably contributed to the enhanced torque-time integral seen most remarkably in the fatigued muscles of the $A B$ group. When comparing the T20-80 in the fatigued state, the VFT is 60 percent shorter than the CFT. The SCI groups did not show the same advantage of the VFT over the CFT in the T20-80 as for the AB group. The T20-80 post-fatigue, when we compared VFT to CFT, was 26 and 37 percent shorter in the $\mathrm{SCl}-\mathrm{A}$ and $\mathrm{SCl}-\mathrm{C}$ groups, respectively (Figure 1).

\section{Torque-Time Integral Over the First $200 \mathrm{~ms}$}

To verify that the greater torque-time integral observed for the VFT is primarily due to a more rapid rise time, we calculated the torque-time integral over the first $200 \mathrm{~ms}$ (TT200) of each contraction (Figure 2). This revealed in the AB subjects an 86 percent greater TT200 for the VFT compared to CFT in the fatigued muscle. The SCI-A and SCI-C groups showed much less enhancement of the VFT over the CFT on TT200: 27 and 41 percent, respectively.

\section{DISCUSSION}

The major finding of this study was that VFTs failed to enhance the torque-time integral in fatigued, paralyzed skeletal muscle. This could be caused by rise times that were already so fast in patients with SCI during CFT stimulation that providing an initial, brief IPI did not reduce the T20-80 or increase peak torque enough to augment the torque-time integral. The TT200 data support this argument, because the advantage of the VFT over the CFT was much smaller in the SCl groups. For the VFT to have an advantage over the CFT, the T20-80 must be shorter for the VFT and/or peak torque higher to counter the fact that the VFT train is $65 \mathrm{~ms}$ shorter than the CFT, as the two trains have the same number of pulses. An analogy can be made to VFT stimulation in fresh muscle of $A B$ subjects. The VFT does not augment the torque-time integral in fresh muscle, because a 60 ms increase in rise time with VFTs is not sufficient to counter the 65 ms longer train duration of the CFT. After fatigue, there is a slowing of contraction, which is made evident by much longer rise times, and the VFT exposes this by enhancing the rise time (by $~ 80 \mathrm{~ms}$ ). This leads to the novel aspect of VFT stimulation, which is an increased torque-time integral despite the difference in train duration ( 20\%, Figure 3).

The contraction rise times of the SCI subjects studied were already so fast that a shorter IPI at the beginning of the train was not sufficient to increase the torque-time integral. Even after fatigue, although there was an 60 percent increase in the T20-80 (compared to pre-fatigue) of SCl subjects, the rise times were still about the same as for fresh muscle of $A B$ subjects and, as previously mentioned, VFTs do not augment torque-time integrals of fresh AB muscle. The post-fatigue VFT T20-80 was about 40 and 100 ms shorter in $\mathrm{SCl}$ and $\mathrm{AB}$ groups, respectively, when compared to their corresponding post-fatigue CFT. It is evident that a $40 \mathrm{~ms}$ reduction is not sufficient to counter the 65 ms longer train duration of the CFT. Therefore, the brief IPI of the VFT was not adequate to counter fatigue in $\mathrm{SCl}$ muscle when the VFT has the same number of pulses as the CFT.

Effect size (Cohen's $d$ ) was calculated for the post-fatigue torque-time integral for VFTs over CFTs for each of the three groups. This measure is often used to imply practical significance to research findings. According to Cohen, a small, medium, or large effect would correspond to ad-value of 0.2 , 0.5 , and 0.8 , respectively [23]. The data in our study suggest that the effect of VFT on the postfatigue torque-time integral was large in the $A B$ group and small in the $\mathrm{SCl}-\mathrm{C}$ group. Our previous studies on $A B$ subjects showed similar findings $[9,10]$. The SCI-A group did not realize a positive effect of VFT stimulation in the post-fatigue states. This suggests that the VFT stimulation as used in this study may not be as effective in $\mathrm{SCl}$ subjects as it is in $\mathrm{AB}$ individuals. 
Might the VFT stimulation be advantageous if the train duration was increased by adding an additional pulse? This could potentially show benefit by increasing the torque-time integral as compared to CFTs, but adding an additional pulse could also increase fatigue. It has been suggested that fatigue might be related to the total number of pulses given; therefore, adding additional pulses to a train may cause more fatigue over time [24]. As Russ and Binder-Macleod point out, adding an additional pulse may also cause more fatigue over time by decreasing the amount of rest between trains [8]. Adding additional pulses to the VFT seems contrary to their potential benefit of reducing muscle fatigue. Another potential manner in which the VFT could be shown to augment the torque-time integral in SCl patients is to shorten the train duration of both the CFT and VFT. For example, if a four-pulse train was used, the CFT would be $210 \mathrm{~ms}$, compared to a $145 \mathrm{~ms}$ VFT. The initial pulse would be relatively more meaningful and a $40 \mathrm{~ms}$ reduction in rise time might significantly enhance the torque time integral for the shortened trains. In terms of clinical electrical stimulation, the problem with having such a short train is that the contraction may not be long enough to perform any functional activities. For example, in a previous study that used dynamic knee extension exercise to increase muscle mass, stimulation current was on for approximately $5 \mathrm{~s}$ at $30 \mathrm{~Hz}$ [21]. Thus, there would be a total of 150 pulses during the contraction, and adding an additional pulse at the beginning to increase the rate of rise in force may not be indicated.

It should be appreciated that $\mathrm{SCI}$ has a dramatic effect on skeletal muscle not equaled by many other conditions. Affected muscles from chronic SCI patients have a higher proportion of fast fibers that are small in size and that are low in enzymes involved in ATP resynthesis, thereby making them highly susceptible to fatigue. While VFT stimulation with the parameters used in the present study failed to show significant benefit in the SCI sample, they may still be useful in conditions not quite as extreme and, indeed, the VFTs have been shown to reduce fatigue in AB subjects. It is also possible that the mechanism(s) of fatigue may not be the same in $\mathrm{SCl}$ and $\mathrm{AB}$ subjects. Castro et al. reported that muscle fatigue was unrelated to differences in the content of enzymes involved in ATP resynthesis [4]. These authors suggested that the fatigue found in SCI patients may be partly due to contraction-induced muscle injury. In fact, short-term unweighting has been shown to increase the vulnerability to exercise-induced muscle injury in humans and lower mammals $[25,26]$. It has also been reported that fibers 6 months post-SCI have mismatched sarco(endo)plasmic reticulum calcium-ATPase and myosin heavy chain isoforms that could influence the fatigability of fibers [27].

There is a report that doublets produced similar responses in $\mathrm{AB}$ subjects as compared to four $\mathrm{SCI}$ patients, but the $\mathrm{SCl}$ patients had been previously conditioned with electrical muscle stimulation (EMS) for a minimum of 6 months [28]. The prior conditioning done by Karu et al. consisted of stimulating the QF muscle group for $1 \mathrm{hr}$ each day, as well as performing FES-assisted cycling 3 days per week [28]. This activity may have had training effects such as converting fast, fatigable fibers to become more fatigue-resistant. It may also have conditioned the muscle so that it was not susceptible to contraction-induced muscle injury, as has been previously shown in unloaded skeletal muscle $[25,26]$. Thus, SCI muscle may need to be conditioned first, by gradually increasing contractile activity over time, prior to the use of VFTs.

\section{CONCLUSION}

This study suggests that VFTs augment the torque-time integral in the fatigued $\mathrm{m}$. QF; this was certainly the case in the $A B$ group and, to a lesser extent, the $\mathrm{SCl}-\mathrm{C}$ group, but it was not the case in the SCI-A group. Because SCl represents one of the most extreme conditions to which human skeletal muscle is subjected, other conditions in which the muscle fibers have not been altered to the same extent may still benefit from these stimulation parameters.

\section{ACKNOWLEDGMENT}


The authors would like to thank the subjects for their participation in this study. Funding was provided, in part, by the Shepherd Center, the Foundation for Physical Therapy, and the National Institutes of Health (HD 37439-S1 and HD 39676).

\section{REFERENCES}

1. Grimby G, Broberg C, Krotkiewska I, Krotkiewski M. Muscle fiber composition in patients with traumatic cord lesion. Scand J Rehabil Med 1976;8(1):37-42.

2. Martin TP, Stein RB, Hoeppner PH, Reid DC. Influence of electrical stimulation on the morphological and metabolic properties of paralyzed muscle. J Appl Physiol 1992;72(4): 1401-16.

3. Round JM, Barr FM, Moffat B, Jones DA. Fibre areas and histochemical fibre types in the quadriceps muscle of paraplegic subjects. J Neurol Sci 1993;116(2):207-11.

4. Castro MJ, Apple DF Jr, Staron RS, Campos GE, Dudley GA. Influence of complete spinal cord injury on skeletal muscle within 6 months of injury. J Appl Physiol 1999; 86(1):350-58.

5. Adams GR, Harris RT, Woodard D, Dudley GA. Mapping of electrical muscle stimulation using MRI. J Appl Physiol 1993;74(2):532-37.

6. Kim CK, Bangsbo J, Strange S, Karpakka J, Saltin B. Metabolic response and muscle glycogen depletion pattern during prolonged electrically induced dynamic exercise in man. Scand J Rehabil Med 1995;27(1):51-58.

7. Binder-Macleod SA, Lee SC, Baadte SA. Reduction of the fatigue-induced force decline in human skeletal muscle by optimized stimulation trains. Arch Phys Med Rehabil 1997;78(10):1129-37.

8. Russ DW, Binder-Macleod SA. Variable-frequency trains offset low-frequency fatigue in human skeletal muscle. Muscle Nerve 1999;22(7):874-82.

9. Slade JM, Bickel CS, Warren GL, Dudley GA. Variable frequency trains enhance torque independent of stimulation amplitude. Acta Physiol Scand 2003;177(1):87-92.

10. Bickel CS, Slade JM, Warren GL, Dudley GA. Fatigability and variable-frequency train stimulation of human skeletal muscles. Phys Ther 2003;83(4):366-73.

11. Burke RE, Rudomin P, Zajac FE. Catch property in single mammalian motor units. Science 1970;168:122-24.

12. Binder-Macleod SA, Barker CB, 3rd. Use of a catchlike property of human skeletal muscle to reduce fatigue. Muscle Nerve 1991;14(9):850-57.

13. Bawa P, Calancie B. Repetitive doublets in human flexor carpi radialis muscle. J Physiol 1983;339:123-32.

14. Maton B, Gamet D. The fatigability of two agonistic muscles in human isometric voluntary submaximal contraction: an EMG study. II. Motor unit firing rate and recruitment. Eur J Appl Physiol Occup Physiol 1989;58(4):369-74.

15. Griffin L, Garland SJ, Ivanova T. Discharge patterns in human motor units during fatiguing arm movements. J Appl Physiol 1998;85(5):1684-92.

16. Hillegass EA, Dudley GA. Surface electrical stimulation of skeletal muscle after spinal cord injury. Spinal Cord 1999; 37(4):251-57.

17. Stevenson SW, Dudley GA. Dietary creatine supplementation and muscular adaptation to resistive overload. Med Sci Sports Exerc 2001;33(8):1304-10.

18. Akima H, Foley JM, Prior BM, Dudley GA, Meyer RA. Vastus lateralis fatigue alters recruitment of musculus quadriceps femoris in humans. J Appl Physiol 2002;92(2): 679-84.

19. Castro MJ, Apple DF Jr, Rogers S, Dudley GA. Influence of complete spinal cord injury on skeletal muscle mechanics within the first 6 months of injury. Eur J Appl Physiol 2000;81(1-2):128-31.

20. Dudley GA, Harris RT, Duvoisin MR, Hather BM, Buchanan P. Effect of voluntary vs. artificial activation on the relationship of muscle torque to speed. J Appl Physiol 1990;69(6):2215-21.

21. Dudley GA, Castro MJ, Rogers S, Apple DF Jr. A simple means of increasing muscle size after spinal cord injury: a pilot study. Eur J Appl Physiol Occup Physiol 1999;80(4): 394-96.

22. Harris RT, Dudley GA. Factors limiting force during slow, shortening actions of the quadriceps femoris muscle group in vivo. Acta Physiol Scand 1994;152(1):63-71.

23. Cohen J. Statistical power analysis for the behavioral sciences. 2nd ed. Hillsdale, NJ: Lawrence Erlbaum Associates; 1988.

24. Marsden CD, Meadows JC, Merton PA. "Muscular wisdom" that minimizes fatigue during prolonged effort in man: peak rates of motoneuron discharge and slowing of discharge during fatigue. Adv Neurol 1983;39:169-211.

25. Warren GL, Hayes DA, Lowe DA, Williams JH, Armstrong RB. Eccentric contraction-induced injury in normal and hindlimb-suspended mouse soleus and EDL muscles. J Appl Physiol 1994;77(3):1421-30.

26. Ploutz-Snyder LL, Tesch PA, Hather BM, Dudley GA. Vulnerability to dysfunction and muscle injury after unloading. Arch Phys Med Rehabil 1996;77(8):773-77. 
27. Talmadge RJ, Castro MJ, Apple DF Jr, Dudley GA. Phenotypic adaptations in human muscle fibers 6 and 24 wk after spinal cord injury. J Appl Physiol 2002;92(1):147-54.

28. Karu ZZ, Durfee WK, Barzilai AM. Reducing muscle fatigue in FES applications by stimulating with N-let pulse trains. IEEE Trans Biomed Eng 1995;42(8):809-17.

Submitted for publication May 15, 2003. Accepted in revised form May 29, 2003. 